\title{
RESEARCH ON THE MODEL OF INTERACTION AND COUPLING RELATIONSHIP BETWEEN STANDARD SYSTEM CONSTRUCTION AND PROCESS MANAGEMENT
}

\author{
Wang Chaofan ${ }^{1}$ and Chen Xinyue ${ }^{2}$ \\ ${ }^{1}$ North China Electric Power University, School of Economics \\ and Management, Wang Chaofan, Beijing, China \\ ${ }^{2}$ Qingdao University, School of Quality and Standardization, \\ Chen Xinyue, Shandong, China
}

\begin{abstract}
The fundamental motivation for enterprises to build a standard system is to meet the subjective needs of the unique use nature of standards, such as benchmarking and criteria, from input to output in the baseline relationship of all their business processes. How to guess the credibility and value of the standard system requires collaborative and mature processes to mediate cognition. Firstly, this paper clarifies the philosophical relationship between standard system construction and process management by using the general system structure theory. Secondly, it systematically summarizes the interaction mechanism between the two from the perspective of methodology. Finally, it designs a conceptual model with the "human regulation" composite system as the core and the coupling between standard system construction and the outer edge of process management, and combed the three mechanism characteristics that affect the fit of the coupling model. In order to provide a new integration idea for enterprise standardization management and process management to jointly realize the optimal value utility.
\end{abstract}

\section{KEYWORDS}

Standardization Discipline, Construction of Standard system, Process Management, Coupling Model.

\section{INTRODUCTION}

The fundamental motivation for an enterprise to build a standard system is to meet the subjective requirements for the unique use of standards, such as benchmarking and standardization, from the input end to the output end in the baseline relationship of all its business processes. The traditional standardization system is structured based on the function as the core, that is, through the in-depth exploration of the manager's management experience and the staff's work experience, the establishment of a corporate standardization system provides a systematic standardization management method and management platform for the realization of corporate strategies (Mai, 2018). This design method will inevitably be affected by the subjective role consciousness of the experience provider and the ideology of standardism, which strengthens the autonomy of the experience provider. It is unavoidable that some experience providers seek to maximize their personal interests or demonstrate their personal indispensability (Wang \& Zhao, 2021). Conceal the individual's unique experience that should be accurately and unreservedly fed back to the standard-setting department as a "secret", or secretly lower the explicit truth threshold David C. Wyld et al. (Eds): CSTY, SIGI, MaVaS - 2021 
of standard items that matches the job ability to invisibly encroach on job resources. This subjective behavior of the experience provider deviates from the original intention of the company to precipitate and reproduce its "skills" when constructing the standard system. At the same time, because internal control is essentially dependent on people, it is difficult to detect and control the scope of the "secret". So that no amount of PDCA cycles in the standardization work will eventually become ineffective consumption. At the same time, if the experience provider suddenly resigns and the successor performs the task with reference to its solidified experience standards, it will be difficult to implement the guidance of the standard, fail to achieve the expected work efficiency, produce repetitive technical labor and knowledge labor, and damage the existing the value utility of the standard system (Krislov \& Samuel, 2020).

How to estimate the credibility and value of the standard system, reduce the risk of enterprise employment, and reduce the degree of dependence on special talents? This requires the introduction of the concept of process management. Process management is a systematic method centered on the standardized structure of end-to-end excellent business processes, and continuously improving the business performance of the organization as the value goal, emphasizing that the content, methods, and responsibilities of the process should be strictly prioritized and statistically controlled. The state is only affected by random factors (Harm Schepel, 2017). Only by controlling the input and process, the quality of the output can be guaranteed, which dilutes the subjectivity and spontaneity of people at work.

In essence, the enterprise standard system is a scientific and organic whole formed by summarizing various standards formulated and implemented around various business processes such as production, operation and management according to their relevance and internal relations (Jaesun Wang \& Seoyong Kim, 2016). Therefore, the effective operation of the enterprise standard system depends on the normal operation of various business processes. This article intends to use general system structure theory to clarify the philosophical relationship between standard system construction and process management, and try to systematically summarize the interaction mechanism between the two from the perspective of methodology. Secondly, the design is based on the "human-regulation" composite system as the core, standard system construction and process. The conceptual model of external coupling of management is expected to provide new integration ideas for the joint realization of enterprise standardization management and process management to achieve the best value and utility.

\section{The Philosophical Relationship between Standard System Construction And Process Management}

A feasible and effective model must be based on the correct philosophical relationship. The correct philosophical relationship between the construction of the standard system and the process management should be recognized as: the construction of the standard system provides a theoretical basis for the effective operation of the process. The standard organization behavioral cognition view provides the source of feedback reform (Smith G, 2015). Go back to GB/T 20000.1's definition of standardization: in order to obtain the best order within the established scope, promote common benefits, establish common use and repeated use clauses, and compile, publish and apply documents for actual or potential problems. At the same time, as mentioned in the previous article, process management is based on the guidance of the logical thinking mode of the enterprise and the existing resource capabilities, and the control of the interconnected or interactive activities that transform input into output (Lawrence Busch, 2011).

From the comparison and analysis of the relationship between the generation and application of the two, the purpose of the construction of the standard system is to provide evidence-based 
guarantees for the consistency of the continuity and quality of the production or service provided by the company. It is not an original factor for the company to generate value-added benefits, but it is an indispensable enhancement factor or improvement factor. Its benefit contribution is attributed to the multi-dimensional influence of standardization in improving enterprise technology, work, management, and hardware. At the same time, it can basically only evaluate the input and output factors related to benefit through the black box method. In addition, the total benefit of the construction of the standard system is the result of the full life cycle time integration of the standardization work; the purpose of process management is to improve the compliance and operability of all management objects in the enterprise that have the ability to execute unified behavior instructions, and the transmission of their behavior directly affects the operation results. Therefore, it is the original factor for the enterprise to generate value-added benefits. It is necessary to carry out internal restraint control and benefit evaluation in a white box manner to ensure that the results of the same behavior awareness are unified within the specified tolerance, and the total benefit is equal to the company's production of products or services. The overall value generated. From this analysis, it is concluded that the construction of the standard system and the process management both contribute to the benefits of the enterprise, and the two overlap in the affected objects. They complement each other in the evaluation of the benefits of the enterprise, and they are in a mutually dependent relationship.

Analyzed from the logical relationship between the outlines of the two expressing corporate management concepts, the construction of a standard system is the "donor" of standardization, and it is a process of subjectively studying standardization theories to arrive at a universally applicable method. The focus is on achieving excellent production with a unified and recognized characteristic relationship. The solidification of formal channels and procedures, the standard system is the standard sub-cluster, which is the objective relationship of enterprise management established based on the subjective recognition of the standardization discipline theory. Once the subjective establishment process is completed, it becomes an objective relationship that cannot be changed at will. The rigid rules consciously abided by within the enterprise are the "theoretical" representatives of the enterprise management philosophy; the process management is the "acceptor" of standardization, which is the objective application of the "benchmark" compliance relationship between the standardized objects in the input and output process. In the process of testing and inspection, a set of processes has a variety of standardized implementation methods and procedures. When carrying out process management, each manager will independently select and sequence the originally independent and uncoordinated sequence activities according to his own experience and background, so that the process management is flexible in operation and is the "practical" representative of corporate management concepts. The outline of the standard system construction is the standard system table, and the outline of the process management is the process operation manual. From this analysis, it is concluded that the standard system construction and process management are the standardized "active side" and the standardized "passive side" respectively, which are opposite to each other.

In summary, it is established that the construction of the standard system and the process management are mutually dependent and opposed to the unity of philosophy (Figure 1). 


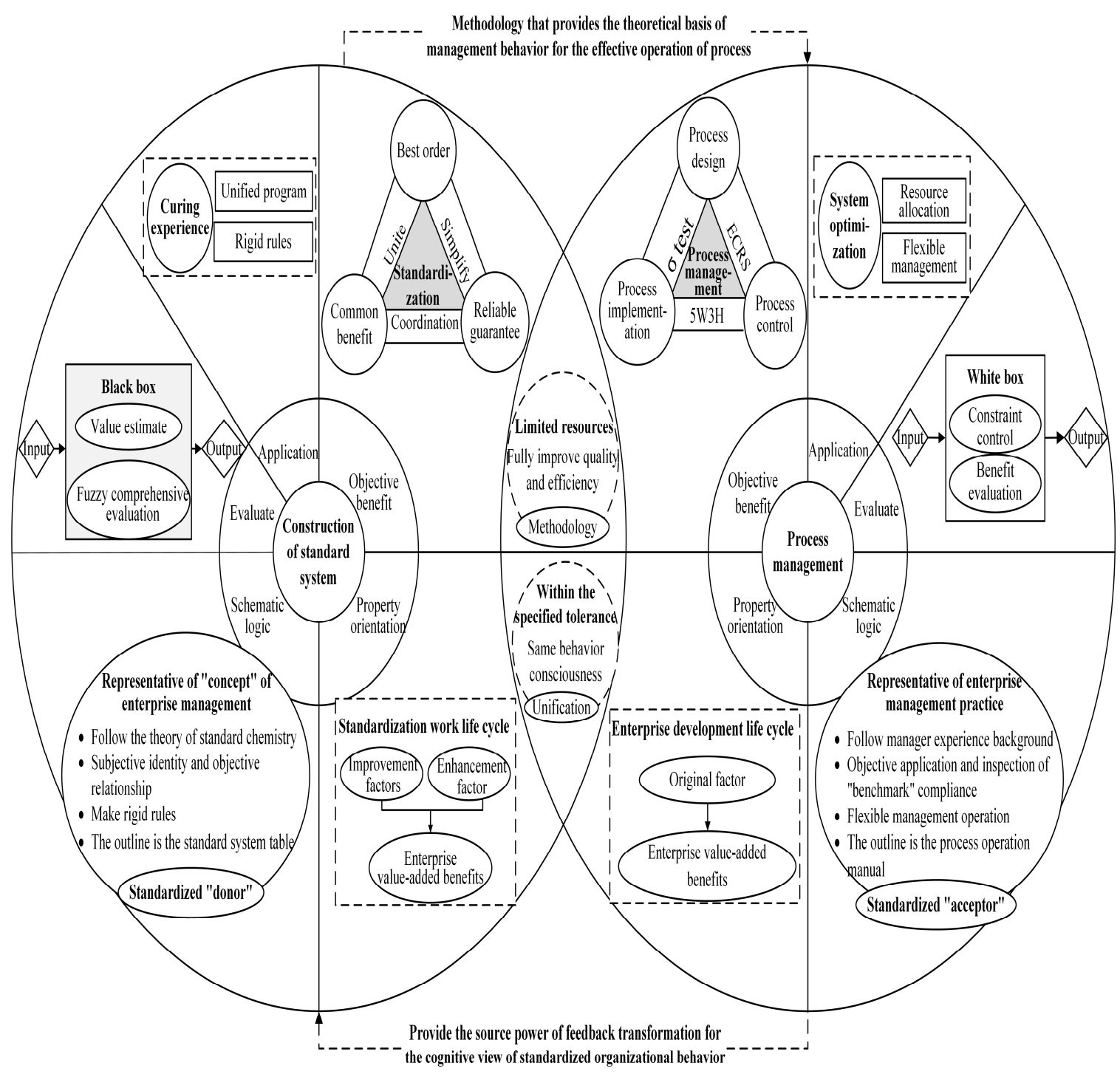

Figure 1. Philosophical relationship between standard system construction and process management.

\section{THE INTERACTION MECHANISM OF STANDARD SYSTEM CONSTRUCTION And Process Management}

\subsection{The impact of standard system construction on process management}

To know the skill is to be round, but to do it straight. Summarizing the role of standard system construction on process management, we might as well express the role of standardization on process management. At the vertical level, because the construction of the standard system is the necessary way and procedure for the implementation of the standardized method of process management, its essence is a standardized application process; in the horizontal scope, the purpose of the construction of the standard system is to organically integrate the standards of the various processes within the enterprise together, to achieve the coordination and unification of process standards and scientific integration, the ultimate goal is to establish the best order of process management for standardization and obtain the "absolute core" service with the best benefits. In summary, the construction of a standard system and standardization have the same 
vertical measurement concept in summarizing the effect of standardization on process management, and the horizontal constraint relationship is consistent. Therefore, the effect of standardization on process management can be extended to express the effect of standard system construction on process management (Figure 2).

First, the role of standardization. When standardization makes the space and time of the process management in a unified state, this unified state makes the process management in a timeinvariant relationship or/and a space-invariant relationship, thereby forming a standardized role in process management or/and a standardized role in space. Timely unification of static process procedures and dynamic process order guarantees the standardized value of process recurrence. The spatial unification of the process constitutes the mutual standardization of different positions in the process. Standardization includes order, and the construction of a recursive standard system plays a role in ordering process management. Ordering includes a spatial order arranged according to the size of the space and a temporal order arranged according to the time sequence. For example, enterprise business processes are staggered, and at the same time, each business process cycle involves the joint participation of more departments, formulating interaction rules that are compatible with the business process and global integration capabilities, which regulates the flow relationship of the process; for complex processes, using standardized modularization concepts to decompose and extract functional programs, unify them into reusable process modules, and then unify the internal logic, status, and functions of the process modules, as well as the external characteristics of the corresponding interfaces, and so on. Standardize the quality behavior of the process module.

Second, the protection function. The safeguard function is a protection and defense function. After the implementation of standardization of process management, the unified state produced will make the specific effects of process management not change with the operator, work time, and location. The maintenance of this state plays a role in protection and defense for the input and output of process management. Therefore, the construction of the standard system has a safeguarding effect. For example: unifying the manufacturing process of products so that the manufacturing process of each product conforms to the unified eligibility requirements can guarantee the quality of each product output; unifying the process of implementing occupational hygiene control can help Different working hours and locations provide equivalent guarantees for the occupational health of the workers.

Third, saving effect. The saving effect of standardization comes from the saving effect brought by the unification of the process. This saving effect has economic saving effect and time saving effect respectively. The economic saving effect is the function of saving process management costs, and the time saving effect is the function of shortening the process time period.

For example, in the relationship between product development and production, the standard system is constructed to unify the specific attributes of similar products, so that products produced according to the same process have a wide range of generalization, thereby increasing the scope and quantity of the same products, and the number of products required. The increase in the production process has led to an increase in the number of applications of the production process, which in turn shared the process design cost and other sunk costs. Therefore, the construction of the standard system has played a cost saving in process management in terms of economic effects.

The saving effect of standardization has also played a driving effect on the efficiency increase of process efficiency. Efficiency is dependent on time, that is, the workload per unit time. The construction of the standard system fixes the best action of production labor. Repetitive labor will drive the improvement of proficiency, shorten the labor time under the same workload and 
improve labor efficiency. Similarly, the increase of process application times in the same time will inevitably improve process application proficiency and process output efficiency. Therefore, the construction of standard system has shortened the process time cycle in terms of time effect.

\subsection{The influence of process management on the construction of standard system}

Process management pursues strict sequence limitation and statistical control from the input end to the output end. It is a continuous activity with a clear starting point and end point to achieve the optimal allocation of scarce resources. The standard system is the top-level design of the standard development, and the expression of the standard relationship system. The construction process of the standard system itself needs to reflect the systematic nature of the standard composition, matching coordination, forward-looking positioning, and advanced content from the input end. Therefore, process management can not only guide the construction of the standard system, but also check the purity of the state of the standard system. The following three points explain the driving effect of process management in promoting the construction of standard systems to realize value:

First, optimize the management of the standard system. Primarily, solve the problem of the integrity and uniformity of the standard. Through process management, all the activities of the production and operation management of the enterprise are determined, the activities and requirements of each node in the entire process are clarified, and the problems of standard-setting departmentalism and multiple issues of the same thing are solved, and it can ensure that the standard is in operation. The division of labor is implemented; secondly, the problem of poor standard coordination is solved. Through process management, the main responsible departments, participating departments, and implementation departments of each node of the process are clarified, and the responsibilities are stipulated in a standard form, which can effectively solve the problem of out of control and control of the joint. Finally, solve the shortcomings of standard compliance and operability. Through process management, all activities from the beginning to the end of each work and business process are defined in the form of flow chart. At the same time, the main responsible departments and personnel, participating departments and personnel, implementation departments and personnel of each node activity, as well as the documents to be executed and the records generated are specified, which can not only meet the requirements of superiors and actual work, And it is easy for the executor to implement.

Second, solve the "systematic degradation" of the standard system. Any standard system is designed based on the development environment, model and technical level at the time. After a period of development, it will become more and more exposed that the structure is not advanced and incomplete, and the standard age is too long, which leads to inapplicability and imprecise standard formulation work. This leads to systemic problems such as duplication, overlap, and contradiction among standards, and process management can reconstruct and optimize them. First of all, process management emphasizes timeliness and conveys a sense of crisis. Process management will monitor the output characteristic deviations in real time during the implementation of the standard. The external influences of the company, such as changes in regulations and policies, and the application of advanced technologies, will obviously lead to a mismatch in the process output interface. Process managers will find out and update the standard system in the first time. Secondly, the process management will review and reflect on the process regularly or irregularly, sort out, collect and rectify the problems encountered in the operation: whether the boundary of the process interface is clear, overlaps or cross-process simplification is feasible, the operability of the process, etc. The regulatory sub-cluster, risk sub-cluster, index sub-cluster, and post sub-cluster of the system are timely perfected and effectively combined. 
Third, realize the flexible allocation of standard system construction resources. The fundamental motivation for the construction of the standard system is to meet people's subjective needs for the unique use nature of the standard. The subjective needs can be divided in order. At the same time, the construction resources of the standard system are scarce and constrained. It is biased to evenly allocate the resources to each module of the construction process of each standard system. Process management will give priority to the process modules that directly or indirectly contribute to the realization of the organization's strategic objectives under the coordination of the different, clear and implied needs and expectations of relevant stakeholders. At the same time, it will respond quickly according to environmental changes and prioritize according to the standard value demand relationship, so as to realize the flexible and reasonable allocation and supply of limited resources.

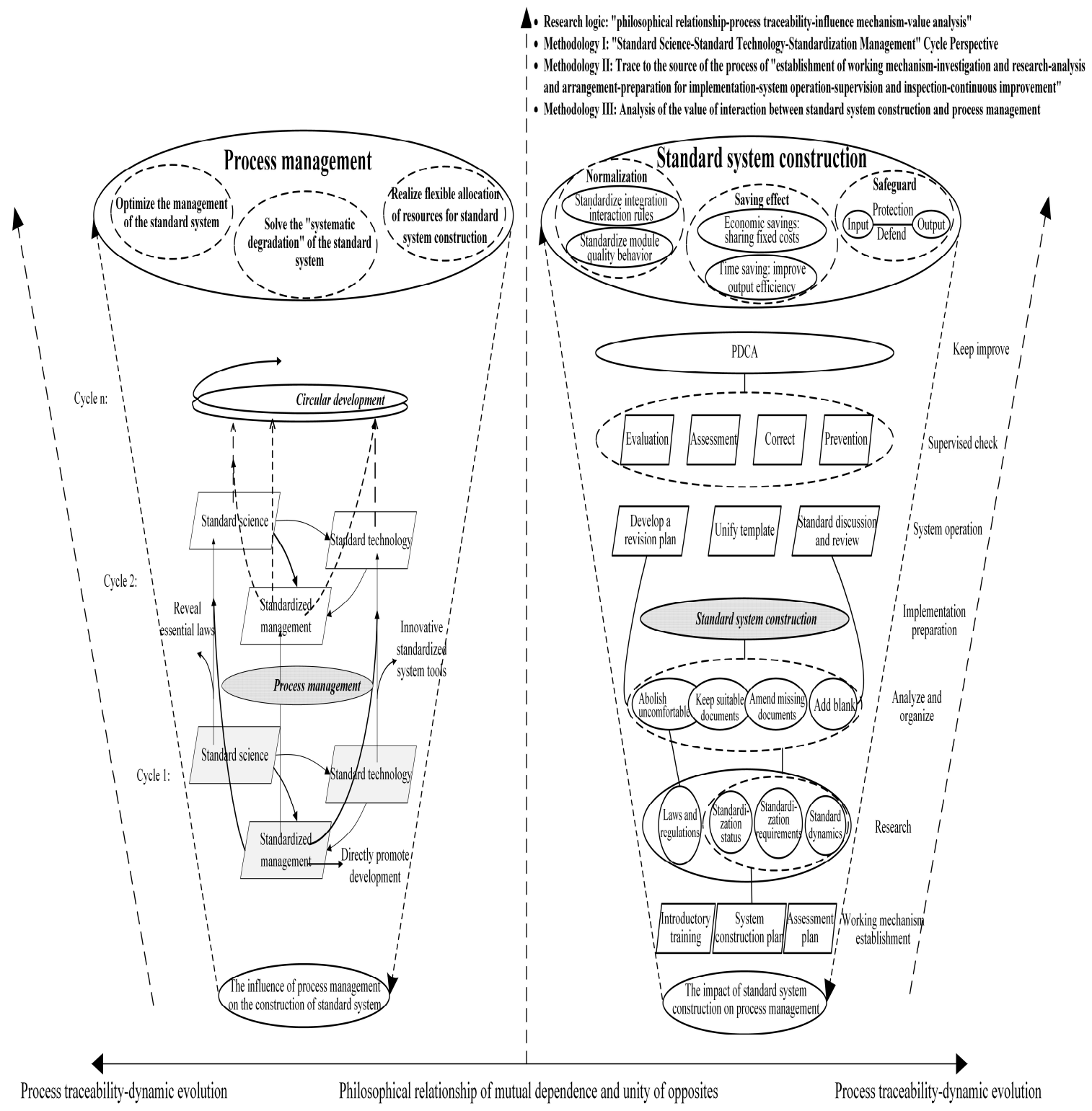

Figure 2. The mutual influence of standard system construction and process management 


\section{Model of INTERACTION AND COUPling Relationship BeTWEen STANDARd System Construction ANd Process Management}

\subsection{Definition of coupling theory}

Coupling theory is used to describe and measure the mutual affinity between two (or more than two) systems with similar properties, which promotes coordination, common development, and dynamic evolution of the systems.

The coupling relationship has three basic characteristics: self-organization, synergy, and measurability. Self-organization means that the coupled system can form a certain structure or function according to a certain rule among the various subsystems in the system without external instructions, so that the system as a whole changes from a chaotic and disordered initial state to a stable and orderly state. Final state evolution; synergy emphasizes the pulling effect of the cooperation between the coupled subsystems and coordination on the formation of the coupling as a whole, that is, in the evolution process of the mutual cooperation and harmonious development of the system, the cooperation of the subsystems makes the overall benefit index of the system "quantitative expansion", subsystem coordination makes the overall structure of the system upgrade "quality improvement"; measurability refers to the orderly development process of the coupled system, the manager can use the data and information that can be obtained to describe the degree of phase change of the coupled structure and the order parameters. The strength and measurability of the synergy between each other enables verification and evaluation of the coupling level among the various subsystems within the system.

\subsection{Standard system construction and process management interaction coupling relationship model composition}

The expression of the coupling relationship between the construction of standard system and process management is different from the expression of the traditional relationship model, because the two are the organic sub-parts of the comprehensive management of the enterprise. The command and coordination of the "platform" of enterprise integrated management, so the expression of the coupling relationship between the two requires a comprehensive and comprehensive analysis of the coordinated changes between standard system construction and process management based on system theory, and the introduction of integrated enterprise management as the third provide the source of the coupling framework.

This paper divides the standard system construction and process management interaction coupling relationship model into a core system with a "people-regulation" composite system as the core, and a supporting platform provided by enterprise integrated management, standard system construction to standardize operating standards, and process management to stimulate endogenous motivation. The external system composed of the three, the components of the core, the external system, and the internal and external systems coordinate and respond to each other, and jointly drive and realize the interaction and coupling of standard system construction and process management (Figure 3 ).

\subsubsection{Kernel system}

The core system of the standard system construction and process management interaction coupling relationship model is a "human-regulation" composite system. Among them, "human" is the behavior subject of coordination and coupling, and "regulation" is the behavior object, which represents the management system to be optimized. 
Acting subject-the participant and beneficiary of the standard system construction and process management. The behavior subject composed of the senior management team, project group, key experts and employees, is in the active position in the "people-regulation" relationship and runs through the transformation process. Motivation acts on "standardization" and "proceduralization", forming a direct driving force for coupling and interaction. Limited by the differences in willingness, resource shortage, and ability range, different actors play different roles in the coupling interaction model: the senior management team coordinates the direction of the coupling strategy through top-level design, path selection and resource allocation; As the basic work group of the enterprise, the project group uses its own cohesion and execution to guide the model coupling interaction and the realization of potential value through organization and coordination. The backbone elites often have a lot of management resources and can provide technology, intelligence and funds for the coupling interaction. Employees are the group most closely connected with the coupling interaction model. They have different requirements for the standard system structure and process maturity at different stages of enterprise development, and their decision-making willingness plays a decisive role in the coordination and interaction of the coupling model. In the process of coupling interaction, the degree of participation of different actors is different, and all parties jointly promote the construction of the standard system and the interaction of process management through the game of interests.

Behavior object-management system to be optimized. The object of behavior to be optimized is not suitable for the current corporate strategic needs or the current use of basic and reasonable institutional documents with potential disadvantages: On the one hand, there are problems such as inefficient use and passive management, and its original normative functions are gradually weakening or even declining. On the other hand, it has good corporate resources and value-added potential, but has not derived other functions through optimized configuration to meet the needs of corporate development.

The core system composed of "people" as the subject of behavior and "regulation" as the object of behavior is functioning well, realizing the dual-driven development of efficient teamwork and excellent systems within the enterprise.

\subsubsection{Outer marginal system}

The outer edge system is composed of many external factors that affect the operation of the core system. In view of the fact that the standard system construction and process management interaction coupling relationship model core system is guided by the integrated management of the enterprise, and there is an intersection of management behaviors between the integrated management of the enterprise and the process management, and the construction of the standard system, it should be placed in the coupling model. The top level of governance, process management, and standard system construction are placed at the grassroots level of policy management.

In the external system, the integrated management of the enterprise is the basic platform for the enterprise to maintain order. It first acts as a medium to realize the connection of resources for the market, upstream suppliers, and downstream distributors, and secondly promotes the quality characteristics of products or services and sustainable production. The organic coordination of the two benefits will finally realize the integration and development of advanced production technology and concepts, and guide the development direction of the internal source system of the coupled interactive model.

Process management is the subsystem that stimulates the endogenous power of the coupled system. It highlights the following three points: First, the redesign of process activities, which 
realizes the reallocation and directional flow of limited resources, promotes the integration of enterprise processes and advanced technologies, and induces the core The behavior subject in the system acts on the driving force of the behavior object to achieve efficient management; the second is the optimization and standardization of process procedures to ensure that the same program is in different time relationships, different spatial relationships, and different operating objects, and can maintain the same presentation of idealized results; The third is the reshaping of the process system, which has caused many changes and transformations in the corporate quality management structure, environmental management structure, and occupational health protection structure, thereby inducing spontaneous changes in the coupled interaction model to a higher level.

As a subsystem of the coupling model, the standard system construction itself has standardization and standardization factors, and has long-term and stability. It is the norm and guarantee for the long-term stable operation of the coupled interactive model. At the same time, the standard system is constructed to build a bridge of mutual trust between the "customer" and the "business". The standard provides the supplier with the product quality requirements that the user expects, and the standard also provides the user with the quality that the supplier provides requirements, provide a basis for inspection and acceptance for both supply and demand parties, provide a basis for supervision of product market management, and realize the integration of mutual trust resources; secondly, the results of the standard system construction are implemented in the standard system table of the text, which is to understand and reproduce the production function of the enterprise The basic roots, and at the same time, it has learned the lessons from the previous enterprise operation and management process, so it is an explicit expression of the precipitation of enterprise experience; in addition, the construction of the standard system has always maintained the basic orientation of continuous optimization and dynamic development, ensuring the coupling model at this stage stability, while promoting the coupling model to a more scientific and efficient stage of improvement.

At the same time, due to the openness of the system, each factor of the subsystem can directly exchange material flow, value flow and information flow with the core system, and indirectly control the interaction direction of the coupling model by influencing the willingness, resources and capabilities of the actors. 


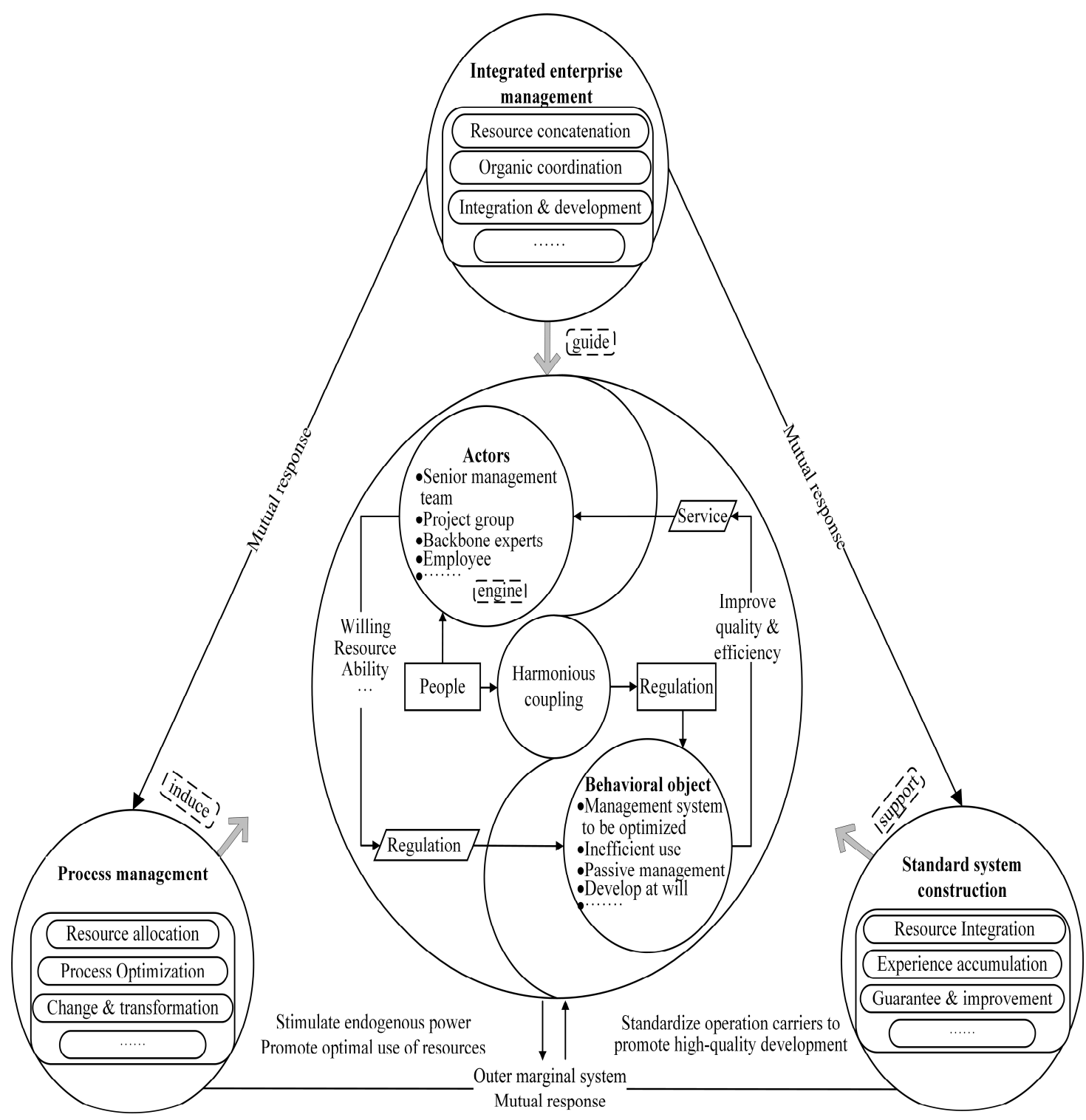

Figure 3. Model of interaction and coupling relationship between Standard system construction-Process management

\section{Three Coupling Mechanism Characteristics}

Analyzed from the perspective of management practice, the construction of standard system is the process of "realizing the unified state of the agreed scope", and the process management is the process of "guaranteeing the value reproduction of the forming sequence module". How to make the two coordinate in the coupling model It is more compatible and the coupling evolution process is smoother. The following is a list of three coupling mechanism characteristics that need attention:

\subsection{The coupled system must be compatible with the dynamic deduction of the subsystem}

Viewing the subsystem as a single system, the construction of the standard system itself is a complete standardization cycle, which is in a state of standardization and optimization at all 
times; process management standardizes and systematically reorganizes and improves the process, while continuously improving the business performance of the organization, that is, it is in a state of process optimization. Both of them are in the process of their own dynamic deduction from this perspective, which also promotes the coupling model to spontaneously transition from a low-level stable stage to an unstable stage, and then develop into a higher-level stable stage.

\subsection{The coupling effect has inertia}

The coupling effect has the nature of state preservation, that is, the preservation ability of coupling "reform". The inertial nature of the coupling effect is embodied in two aspects. On the one hand, the coupling state is not easy to disappear; on the other hand, the coupling state is not easy to establish. The characteristics of the inertial nature of the coupling effect are: the inertial nature of the coupling effect is universal. As long as it is the state of the coupling effect, it must be a state of inertia; the magnitude of the coupling effect inertia is proportional to the coupling range and the time period of the coupling. The larger the coupling range and the longer the forming time period, the greater the inertia of the coupling state, and vice versa.

\subsection{Use "system effect" to evaluate coupling benefits}

First of all, the coupling benefit is the benefit produced by the application of the coupling concept. Coupling benefit is not the input and output relationship of the economic value of the coupling model, but the input and output relationship of the coupling concept. The benefit of the coupling model is not actually the contribution benefit of the model itself, but the benefit of applying the coupling concept and bringing about changes. The establishment of a coupling state requires all-round investment in personnel training, equipment, equipment, environment, and materials. From the perspective of knowledge, the coupling concept is a body of knowledge, not an economic reproductive body. It is not appropriate to use investment standards to evaluate the benefits of coupling; do not think too much about the best indicators of the sub-system, because the view of system science believes that the best sum of the sub-systems is not equal to the best total system, because the realization of the overall goal will bring "system effect", that is, the effect generated by the system as a whole exceeds the sum of the effects of various parts of the system.

\section{REFERENCES}

[1] Modern Chinese Dictionary, (2017) The Commercial Press, Dictionary Editing Office, Institute of Language Studies, Chinese Academy of Social Sciences,.

[2] ISO 9000 family, Quality management.

[3] Mai Lvbo, (2018) The method of standard system optimization. China Standardization, (07): 58-65.

[4] Wang Chaofan, Zhao Chaofei, (2021) Research on the construction of enterprise standard system from the perspective of methodology . China Standardization, (01): 90-94.

[5] GB/T 13016-2018, Principles and Requirements for the Construction of Standard System.

[6] Krislov, Samuel, (2020) How nations Choose Product Standards and Standards Change Nations, Pittsburgh: University of Pittsburgh Press.

[7] Harm Schepel, (2017) The Constitution of Private Governance: Product Standards in the Regulation of Integrating Markets, Oxford and Portland, Oregon.

[8] Jaesun Wang, Seoyong Kim, (2016) Time to get in: The contrasting stories about government interventions in information technology standards (the case of CDMA and IMT-2000 in Korea), Government Information Quarterly, 24.

[9] Smith G, (2015) Interaction of Public and Private Standards in the Food Chain, OECD Food, Agriculture and Fisheries Papers, No. 15, OECD publishing.

[10] Lawrence Busch, (2011) Standards: Recipes for Reality,Cambridge, MA and London: The MIT Press. 


\section{AuThors}

Wang Chaofan is a postgraduate of management at North China Electric Power University, and currently serves as the general manager of Bohai Quality and Standardization Service Company.

(C) 2021 By AIRCC Publishing Corporation. This article is published under the Creative Commons Attribution (CC BY) license. 